Classification

Physics Abstracts

$47.55 \mathrm{M}$

\title{
Multiple scale structure of non wetting fluid invasion fronts in 3D model porous media
}

\author{
E. Clément, C. Baudet and J. P. Hulin \\ Laboratoire d'Hydrodynamique et de Mécanique Physique, E.S.P.C.I., 10 rue Vauquelin, \\ 75231 Paris Cedex 05, France
}

(Reçu le 17 juillet 1985, accepté sous forme définitive le 18 octobre 1985)

\begin{abstract}
Résumé. - Nous présentons des résultats de mesures expérimentales de la structure de fronts d'invasion obtenus par une injection verticale lente de métal de Wood non mouillant dans un massif de verre pilé non consolidé. Nous analysons la géométrie de coupes du front d'invasion perpendiculaires et parallèles à la direction de l'écoulement. La structure des coupes perpendiculaires est autohomothétique dans une gamme d'échelles de longueur de 1 à 20 avec une dimension fractale de 1,65. Les profils de saturation obtenus à partir de coupes parallèles à l'écoulement sont qualitativement en accord avec les modèles de percolation. Nous discutons différentes prédictions théoriques sur la géométrie des coupes perpendiculaires à l'écoulement.
\end{abstract}

\begin{abstract}
We report experimental measurements on the structure of invasion fronts obtained by slow vertical injection of liquid, non wetting, Wood metal into non consolidated crushed glass. The geometry of plane cuts of the invasion front both parallel and perpendicular to the flow direction is analysed. The structure of perpendicular cuts is self similar in a length scale range of 1 to 20 with a fractal dimension 1.65. Saturation profiles measured from parallel cuts are in qualitative agreement with invasion percolation models. Possible theoretical predictions for the geometry of horizontal cuts are discussed.
\end{abstract}

\section{Introduction.}

2 phase flow in porous media is a subject of large present interest because of its practical applications in the oil industry (oil-water flows in oil reservoirs) and in hydrogeology (air-water flows in partly saturated soils).

While this topic has greatly benefited from recent theoretical developments of scaling approaches [1-4] to the physics of porous media, very few experiments were performed in this perspective.

Lenormand $[5,6]$ has analysed the low velocity invasion of $2 \mathrm{D}$ horizontal micromodels by a non wetting fluid (drainage). He showed that a minimum threshold injection pressure is necessary to invade the bulk of the micromodel (breakthrough point). At this threshold, the invading fluid volume has a fractal geometry analogous to a $2 \mathrm{D}$ bond percolation cluster. He measured a fractal dimension $d_{\mathrm{f}} \simeq 1.80-1.83$ in excellent agreement with " invasion percolation » numerical simulations [3,9] taking into account trapping effects for an incompressible displaced wetting fluid. For classical percolation with no trapping effects, $d_{\mathrm{f}}$ is slightly higher $(\simeq 1.89$ in $2 \mathrm{D})$. 
Recently, measurements on a non consolidated glass column initially saturated with a matched index water- $\mathrm{ZnCl}_{2}$ solution and emptying under gravity have been reported by Jacquin [7].

In this Letter, we present experimental results obtained on a 3D model system (non consolidated crushed glass) slowly invaded by a low melting point, non wetting, liquid alloy (Wood metal) used as a liquid phase (Fig. 1). After the injection, the alloy is solidified and plane cuts of the invasion front are performed both parallel (Fig. 2) and perpendicular (Fig. 3) to the flow.

We analyse both the fluid saturation profile and the cut geometry in the light of the invasion percolation models. These describe well low velocity injections when viscous forces can be neglected; however, we have to take into account in addition the effect of hydrostatic pressure gradients (the injection is vertical).

The first experiments have been performed by one of us (E.C.) [8] together with Jacquin (I.F.P. laboratories) who introduced us to the technique of Wood metal injection also developed by Bourbié and Zinzner (I.F.P.). Subsequent samples such as that of figure 2 were obtained in our laboratory.

\section{Experimental procedure and set-up (Fig. 1).}

2.1 INJECTED FLUID. - Wood metal has been chosen because it is a low melting point alloy $\left(T_{\mathrm{m}} \simeq 70^{\circ} \mathrm{C}\right)$. It can be slowly solidified after the injection in the liquid state in order to examine the invasion front structure. Wood metal, like mercury, also does not wet the glass : therefore parasitic effects due to poorly defined wetting characterics are minimized (particularly while the alloy moves into the material). However, the high Wood metal density induces large gravitational effects which must be taken into account.

2.2 Porous MATERIAL. - We inject the liquid Wood metal into non consolidated crushed glass : this allows us to remove the glass grains from the non invaded parts after the alloy solidification in order to observe the invasion front. The granulometry is carefully selected between mesh sizes of 200 and $225 \mu \mathrm{m}$ in order to avoid stratification while filling the tube.

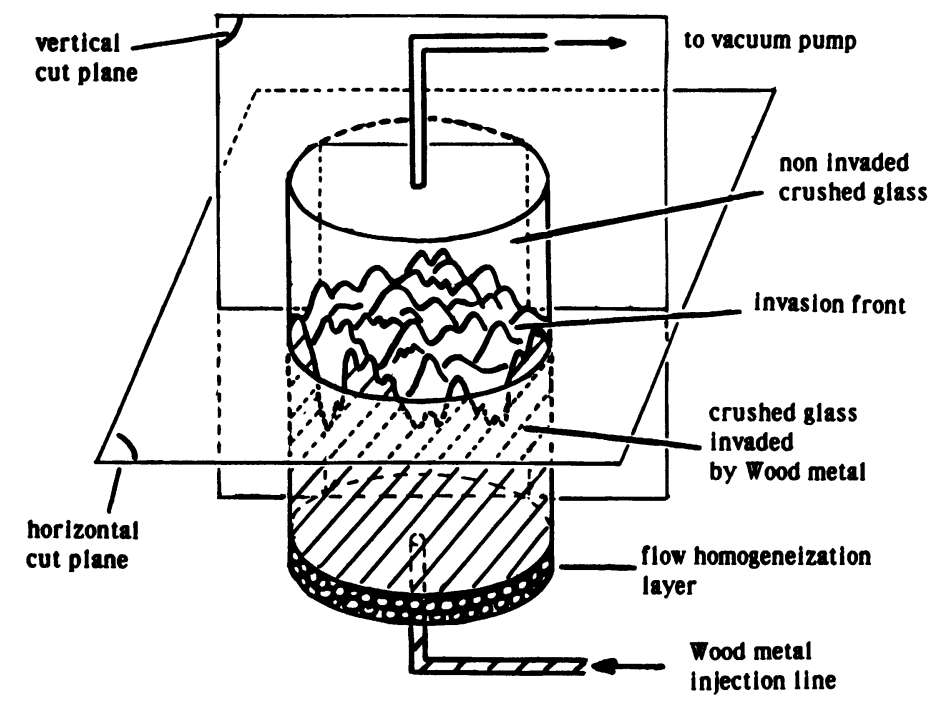

Fig. 1. - Schematic view of experimental set-up for injecting liquid Wood metal into non-consolidated crushed glass : an injection line connects the bottom of the injection volume to a storage tank (not shown here) initially filled with the alloy. The whole set-up is contained inside a heated oven. 


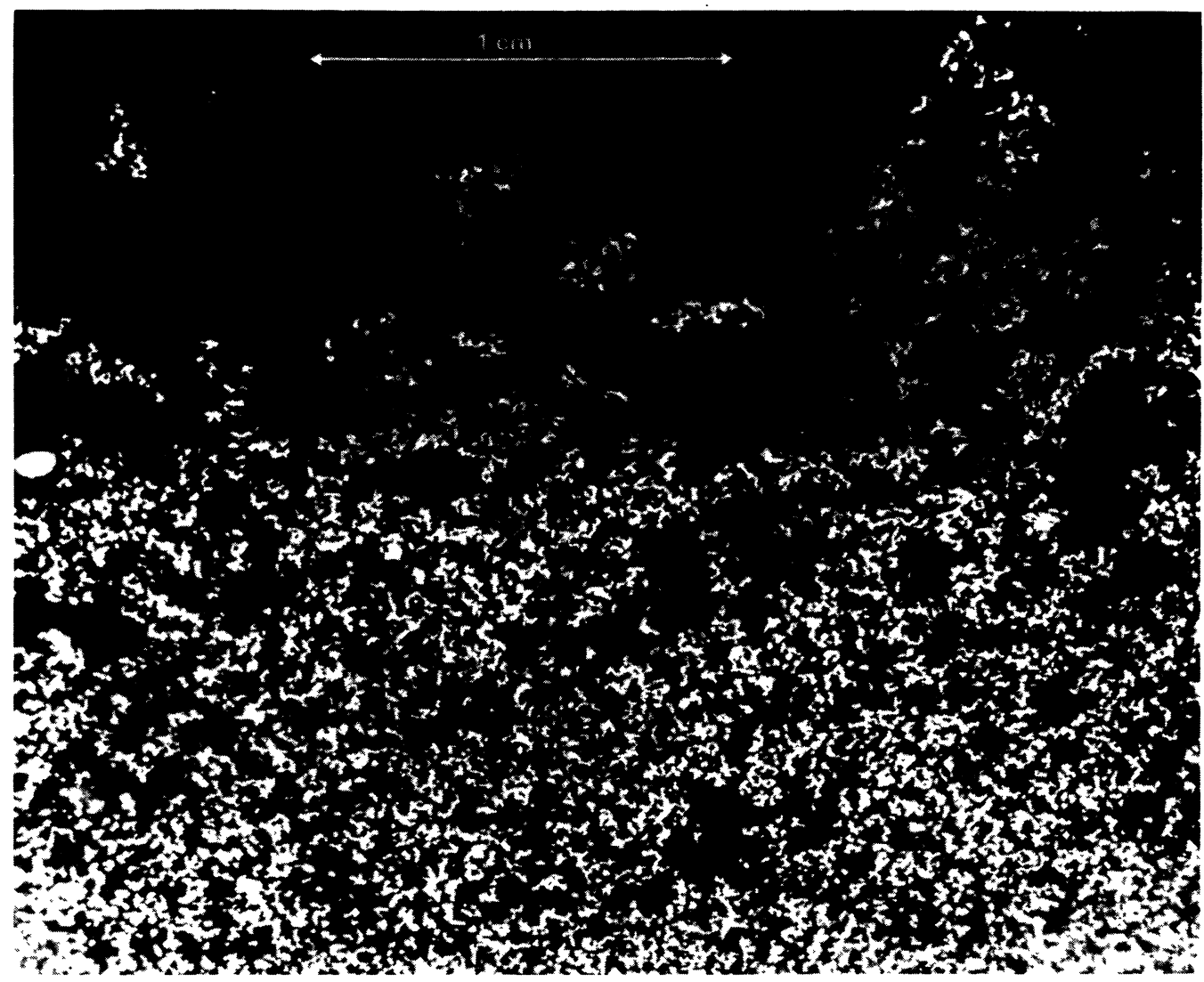

Fig. 2. - Vertical cut parallel to the flow direction of a structure obtained in our laboratory by a very slow injection of Wood metal into non consolidated crushed glass. The invasion front has been potted in an epoxy resin to consolidate it. The Wood metal grains appear as bright points due to their high reflectivity.

We chose crushed glass instead of microspheres because it gives a wider pore size distribution and a geometry closer to that of real rocks.

We inject the Wood metal uniformly at the bottom of a vertical tube $(15 \mathrm{~cm}$ high, $10 \mathrm{~cm}$ inside diameter) filled with the glass powder and kept under vacuum in order to avoid trapping effects during the injection.

2.3 INJECTION PROCEDURE. - The injection set-up is inside a thermally regulated oven in order to avoid temperature gradients. Initially, Wood metal is inside a storage container connected to the porous volume by an injection tube (Fig. 1).

A low injection flow-rate is obtained by slowly raising the container 0.5 by $0.5 \mathrm{~mm}$, a length of the order of the grain size. The typical total duration of the injection is 48 hours. In this way, the level difference between the container and the porous medium remains just sufficient to overcome the capillary forces on the front. The flow velocity is always small in order to keep viscous forces negligible. We checked experimentally that moving the outside level very slowly is a key factor for obtaining a maximum vertical extent of the invasion front and reproducible results. 


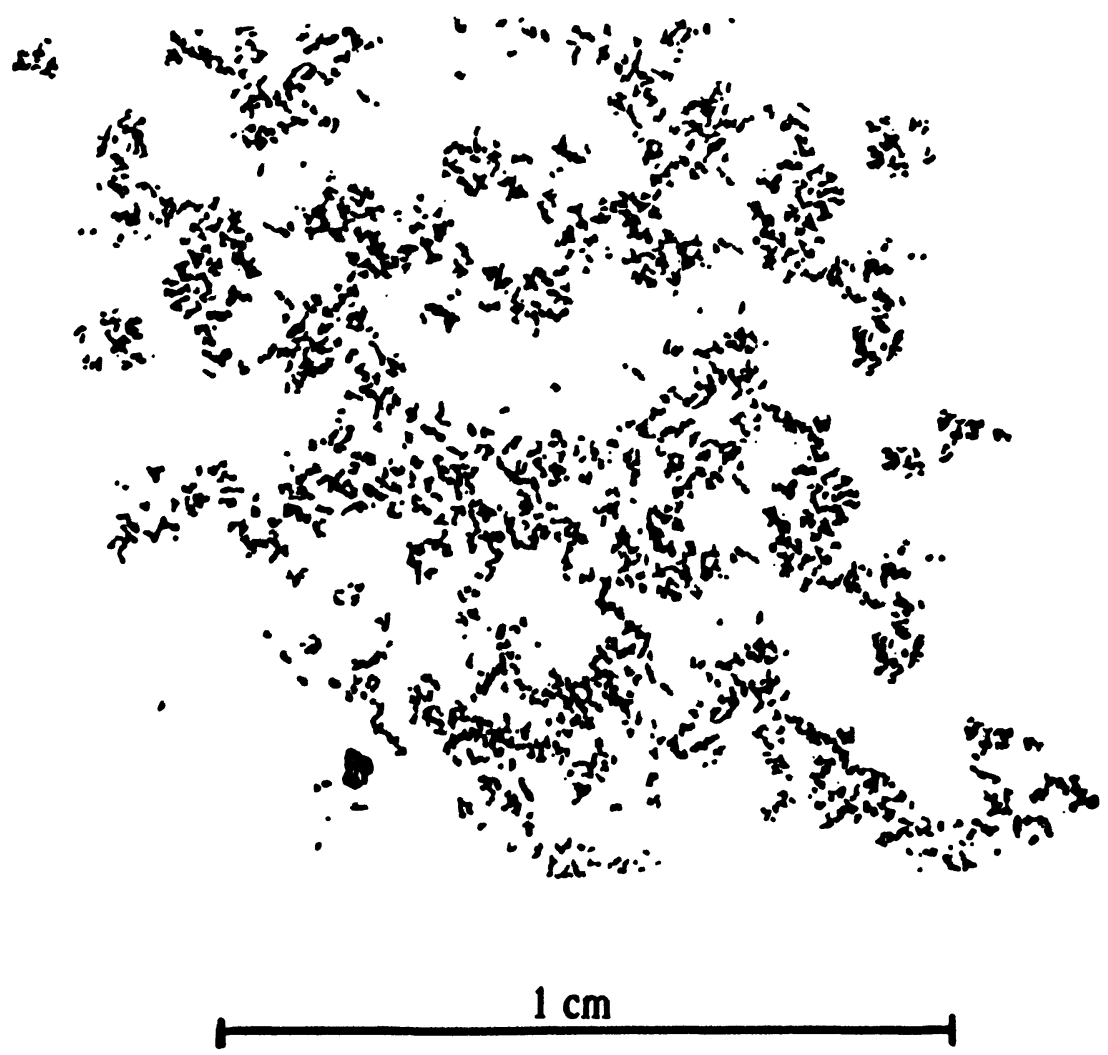

Fig. 3. - Horizontal cut perpendicular to the flow direction of the invasion front obtained by injection of Wood metal into crushed class. In this picture, the Wood metal grains appear as dark points. The injection and sample preparation have been realized at I.F.P. by T. Bourbié and C. Zinzner.

After the injection, the Wood metal is solidified very slowly, keeping the temperature and pressure uniform. In this way, we avoid curvatures of the invasion front due to a non uniform solidification of the metal.

2.4 ANALYSIS OF THE EXPERIMENTAL RESULTS. - After the non invaded glass has been tossed-off, the invasion front is clearly visible and can be examined to detect possible stratifications. Then, the front is potted in epoxy resin under vacuum to avoid mechanical degradation and cuts parallel (vertical) and perpendicular (horizontal) to the flow are performed (Figs. 2 and 3).

The cuts are machined and polished with coarse sand-paper in order to keep the Wood metal grains to their real size and avoid their blurring by an epoxy layer. The samples are photographed with a lighting making use of the high reflectivity contrast between Wood metal and epoxy resin; these pictures are then digitized and analysed on a Victor S1 computer.

\section{Analysis of the invasion front geometry.}

3. 1 TheORETICAL PREDICTIONS ON. THE INVASION. FRONT STRUCTURE.

3.1.1 Invasion percolation model in zero gravity. - The porous medium is pictured as a lattice of pores connected by narrower throats. Slow invasion by a non-wetting fluid is described as a sequence of motions of individual interface menisci from one pore to another through a throat. A key notion is the accessibility of an individual pore throat at a given injection pressure $\Delta p_{\text {inj. }}$. 
A meniscus at the boundary of the injected non-wetting fluid can pass through a channel of minimum radius $r_{\mathrm{m}}$ between two pores only if $\Delta p_{\mathrm{inj}}$ is larger than the capillary pressure value $\Delta p_{\text {cap }}$ when the meniscus reaches the narrowest section of the channel with :

$$
\Delta p_{\text {cap }}=2 \gamma \cos \theta / r_{\mathrm{m}}
$$

( $\gamma=$ superficial tension, $\theta=$ wetting angle).

At a given fixed injection pressure, the fluid fills up all pores connected to the injection surface by a continuous (although incomplete) lattice of accessible channels.

Under zero gravity conditions, the statistics of the invading fluid distribution is the same as for bond percolation on the lattice of same topological structure where all non-accessible bonds are taken as «cut " bonds. Then, the breakthrough point corresponds to a percolation threshold where the invading fluid has a fractal structure extending through the whole medium.

Differences with classical percolation exist if the porous material is initially filled with a non compressible fluid. Displaced fluid regions surrounded by the injected fluid get trapped and cannot be displaced thereafter (particularly in 2 dimensions). As noted before, this reduces the invading fluid volume at breakthrough as well as the corresponding fractal dimension $[9,10]$.

3.1.2 Influence of gravity [11]. - Hydrostatic pressure variations create a vertical gradient of the effective injection pressure $\Delta p_{\text {inj }}$ driving the meniscus through the pore throats. Therefore, the percentage of pores invaded by the Wood metal decreases with the vertical height $z$ above the injection surface (Fig. 2).

Let us define the total width $\Delta h$ of the invasion front as the vertical distance between the upper edge of the invasion front above which no pores get invaded and the lower limit below which the displaced phase (or the empty volume) is not continuous. Inside the front width $\Delta h$, both the injected and the displaced phase are continuous (biconnexity). If $\Delta r_{m}$ is the width of the minimum radii distribution for the throats, one can estimate an upper bound to the front width $\Delta h$ by :

$$
\Delta h \sim 2 \gamma \cos \theta \Delta r_{\mathrm{m}} /\left(\rho g r_{\mathrm{m}}^{2}\right)
$$

(where $\rho$ is the fluid density).

Relation (2) simply reflects the variations of capillary pressure due to the spread in the pore sizes and should apply exactly to an array of independent vertical tubes with variable radii.

In our experiments, the observed value of $\Delta h$ does not get larger than about $4 \mathrm{~cm}$ due to the high Wood metal density and the structure of the invaded volume is different from a $3 \mathrm{D}$ percolation cluster.

3.1.3 Horizontal cuts and fractal geometry. - Experimentally, we perform invasion geometry analysis in horizontal planes where the local injection pressure and, therefore, the percentage of accessible throats remains constant. Depending on the distance of the cut to the upper edge, we observe either only isolated patches of Wood metal or an " infinite cluster " of invaded pores extending through the whole cut plane : both types of structures belong to a single $3 \mathrm{D}$ cluster.

There are two ways to analyse the invaded zone geometry :

1. Consider the distribution as analogous to $2 \mathrm{D}$ percolation one with a percolation parameter decreasing with the cut plane vertical level. In this approach, clusters of invaded pores all connected together should have a geometry close to $2 \mathrm{D}$ percolation clusters with a fractal structure up to the correlation length or to their size. At the 2D percolation threshold, the fractal dimension should be close to $1.89[9,10]$ (no trapping effects occur because the porous material was initially evacuated). However, this dimension depends on the value of the effective percolation parameter compared to the threshold, and, therefore, on the value of $\Delta z$ for the transverse cut plane [14].

On the other hand, the global invaded area in a $2 \mathrm{D}$ horizontal section differs from a $2 \mathrm{D}$ percolation system : only clusters of accessible pores that are connected to the injection plane get filled 
with metal. In addition, these clusters are not located at random : spatial correlations occur due to connections in the third dimension.

2. In the second approach, the cut is considered as a section of an infinite, locally isotropic, 3D cluster. If we assume that the percolation model is valid, this $3 \mathrm{D}$ cluster has a fractal dimension $d_{\mathrm{f} 3 \mathrm{D}} \simeq 2.5$ at the percolation threshold. Then the global cut structure including all finite and infinite invaded zones should have a fractal dimension $d_{\mathrm{f}}=d_{\mathrm{f} 3 \mathrm{D}}-1 \simeq 1.5$.

Experimentally, it is not possible to determine whether a set of invaded pores belong to the same cluster since the connecting channels are indeed generally hidden by the solid matrix. Hence, we have chosen to analyse the distribution of all pores invaded by Wood metal in the section without attempting to determine whether they are located on a same 2D cluster. We can therefore compare the results only to the predictions from the second approach discussed above.

3.2 EXPERIMENTAL MEASUREMENTS ON PLANE CUTS. - A widespread definition of the fractal dimension for a cluster of identical particles uses the correlation function $C(r)$ for their probability distribution [12]. $C(r)$ represents the probability to find a particle at a given point located at a distance $r$ from another particle. For a fractal structure :

$$
C(r) \propto r^{d_{\mathrm{f}}-2}
$$

$\left(d_{\mathrm{f}}=\right.$ cluster fractal dimension).

We adapted this definition by digitizing the plane cut images with a pixel size $\varepsilon$ smaller than the grain diameter and a $256 \times 256$ resolution. All pixels corresponding to a digitized signal larger than a threshold value are considered as occupied by Wood metal and the others as empty (the threshold is chosen from an histogram of the video signal amplitude).

Practically, we count the number $n(r)$ of occupied cells located between squares of sides $2 r$ and $2(r+\varepsilon)$ centred on an invaded cell. The usual definition would use circles of radii $r$ and $r+\varepsilon$ but, for a self-similar structure, both methods give the same results. Effects associated with the finite size of the image are reduced by using a procedure already applied elsewhere [13] to the analysis of diffusion limited aggregation clusters ; for a given origin site, $n(r)$ is only computed for $r$ values such that the square of side $2 r$ entirely fits within the image boundary.

The results obtained for all possible centre sites are then averaged $: C(r)$ is proportional to $n(r) / r$ where $n(r)$ is the average computed above and the $1 / r$ factor takes into account the increase of the number of potential occupied sites with the distance.

Figure 4 shows the correlation function $C(r)$ obtained for the global plane cut of figure 3; different domains of variation appear :

- for $r \leqslant R$ (size of the largest grains in the porous material), the correlation function variation is related to the microscopic structure of the medium.

- for $r>\xi \simeq 17 R, C(r)$ becomes almost constant and the structure appears as uniform. This may mean that $\xi$ represents the correlation length for the Wood metal 2D structure. Finally, at still larger $r$ values, $C(r)$ drops rapidly as $r$ gets close to the size of the image.

- for $R<r<\xi$ we expect to have a self-similar structure : the variation of $C(r)$ is indeed well described by a power law (Fig. 4). A best fit procedure gives a value $-0.35 \pm 0.05$ for the exponent describing the variation of $C(r)$ in that region. The corresponding fractal dimension is $d_{\text {f exp }}=1.65$, somewhat higher than the value 1.5 expected for the $2 \mathrm{D}$ section of an infinite $3 \mathrm{D}$ percolation cluster. However, in the present experiment, the characteristic dimensions of the invasion front parallel to the flow are not large with respect to those perpendicular to it; the isotropy assumption does not therefore apply at large distances : this may explain the discrepancy with the theoretical $d_{\mathrm{f}}$ value. 


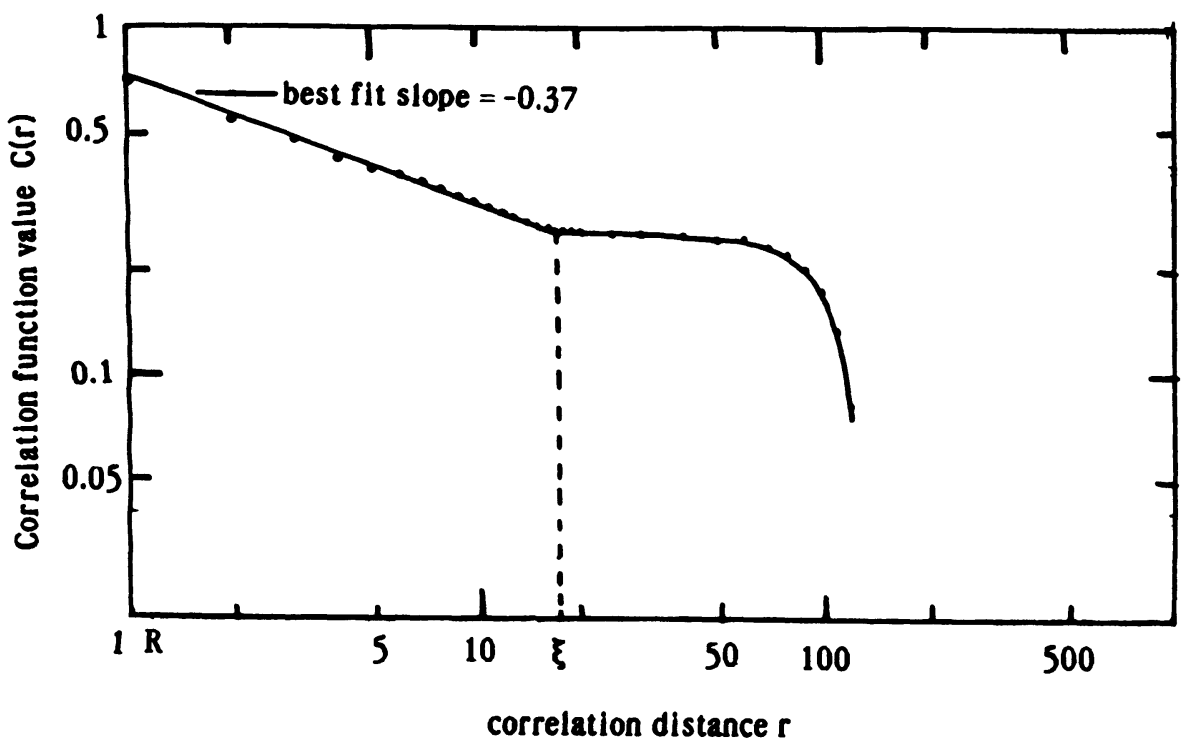

Fig. 4. - Correlation function between the position of the Wood metal grains in the horizontal plane cut of figure 3. The unit length $r=1$ corresponds to the size $R$ of the largest grains.

\section{Vertical variation of the invading fluid saturation.}

4.1 EXPERIMENTAL SATURATION PROFILE MEASUREMENTS. - The saturation profile has been obtained from a cut performed parallel to the vertical flow direction. Using the same digitization and thresholding procedure as in 3.2, an estimation of the saturation profile $S_{\mathrm{nw}}(\Delta z)$ is obtained by counting the number of « occupied " cells at a given height $\Delta z$ below the upper edge (in this section, $\Delta z$ will be taken as $>0$ below the edge).

Figure 5 shows the variation of the local percentage of « occupied » cells with $\Delta z$ in a vertical plane cut parallel to the flow.

4.2 TheORETICAL PREDICTIONS. - Let us first recall the predictions of the invasion percolation model which should only apply in zero gravity and at very low flow-rates where the viscous forces are completely negligible.

Let us define the non wetting fluid saturation $S_{\text {nw }}$ as the fraction of invaded pores. The injection pressure $\Delta P_{\text {inj }}$ controls the fraction of accessible connection channels between pores and can be used as a percolation parameter. Then, one should have :

$$
S_{\mathrm{nw}} \propto\left(\Delta P_{\mathrm{inj}}-\Delta P_{\mathrm{t}}\right)^{\beta}
$$

where $\beta$ is the classical 3D percolation exponent relating the percentage of sites on the infinite cluster and the distance of the percolation parameter above the percolation threshold pressure value $\Delta P_{\mathrm{t}}$.

In a finite gravity, the local value of $\Delta P_{\text {inj }}$ varies linearly with the vertical height; let us first assume that relation (4) remains locally valid and that $\Delta P_{t}$ corresponds to the injection pressure at the upper edge of the invasion front. Then, if $\Delta z$ is the distance to this upper edge, one should expect :

$$
S_{\mathrm{nw}} \propto\left(\Delta P_{\mathrm{inj}}-\Delta P_{\mathrm{t}}\right)^{\beta} \propto \Delta Z^{\beta}
$$




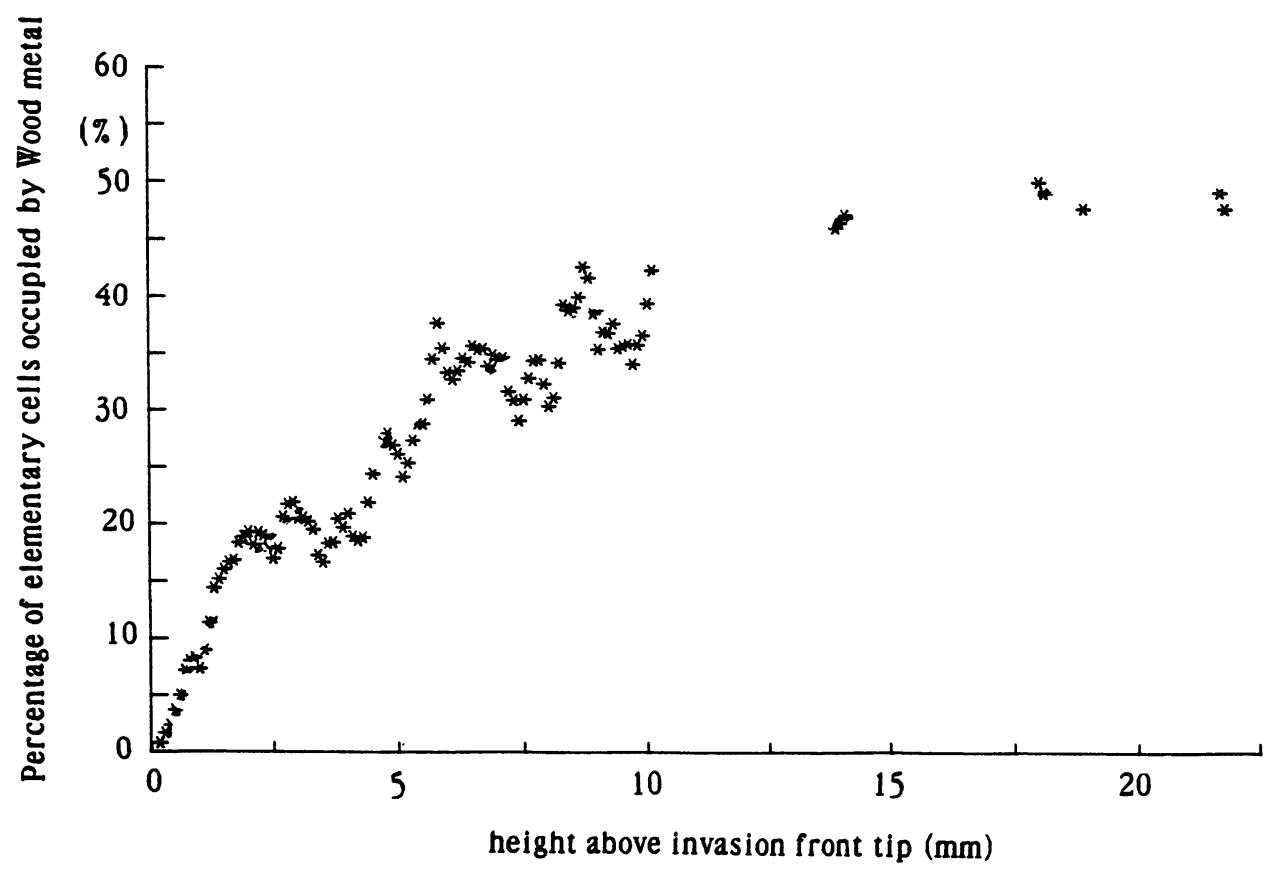

Fig. 5. - Saturation profile obtained from a vertical cut parallel to the flow of an invasion front of Wood metal into crushed glass. The curve corresponds to a sample prepared by T. Bourbié and C. Zinzner and similar to that of figure 3 .

with $\beta \simeq 0.4$ in $3 \mathrm{D}$. This approximation will only be valid for $\Delta z$ values such that $S_{\mathrm{nw}}$ varies slowly at a scale of the order of the correlation length. This condition is not verified at the edge of the front because of the strong gravity effect : the front becomes then broader and the $\Delta z^{\beta}$ law will be distorted. The profile in figure 5 has a sharp edge, in qualitative agreement with equation (5) but the uncertainty is too high to attempt a quantitative $\beta$ determination.

\section{Conclusion.}

We have reported experimental results on the slow invasion of non consolidated crushed glass by liquid Wood metal in qualitative agreement with invasion percolation.

We observed in a horizontal cut of the invaded structure a significant length scale domain where a self-similar description is valid. The measured fractal dimension $d_{\mathrm{f} \text { exp }}=1.65$ is higher than the value corresponding to the cut of a 3D isotropic cluster(1.5).

Further experiments for cut planes at various distances from the front upper edge are in progress to analyse the influence of the local Wood metal saturation on the self-similar properties of the structure.

We have also performed measurements of the invading fluid saturation variation with the vertical distance from the invasion front edge : again, the results agree qualitatively with invasion percolation predictions [15] but it is difficult to evaluate the influence of gravity; we shall repeat the experiment using crushed glass with a smaller grain diameter in order to decrease the relative influence of gravity compared to the capillary forces.

On another hand, more classical models [16] also predict a sharp front edge at finite capillary numbers : the influence of viscosity on the results of 3.5 will be analysed by performing injections at several different constant flow-rates, and/or by saturating initially the medium with a viscous fluid. 


\section{Acknowledgments.}

We wish to thank E. Charlaix, E. Guyon and C. Leroy for many fruitful discussions and suggestions.

We have greatly benefited from the help of T. Bourbié, C. Jacquin and B. Zinzner at I.F.P. who introduced us to the Wood metal injection techniques and provided us with many data and experimental assistance.

One of us (E.C.) has been supported by a grant from the Schlumberger Doll Research Center.

\section{References}

[1] De Gennes, P. G. and Guyon, E., J. Mec. 17 (1978) 403.

[2] Chatzis, J. and Dullien, F. A. L., J. Can. Pet. Tech. 97, Jan-Mar (1977).

[3] Chandler, R., Koplik, J., Lerman, K. and Willemsen, J., J. Fluid Mech. 119 (1982) 249.

[4] Guyon, E., Hulin, J. P. and Lenormand, R., Annales des Mines, special issue « Ecoulements dans les milieux fissurés », 191, 5-6, 17 (1984) (in French).

[5] Lenormand, R., Zarcone, C. and SarR, A., J. Fluid Mech. 135 (1983) 337.

[6] Lenormand, R. and Zarcone, C., to be published in Phys. Rev. Lett. (1985).

[7] JaCQuin, C., C.R. Acad. Sci. 300, série II, no 15 (1985).

[8] Groupe Poreux PC, in Proc. de l'Ecole d'Hiver des Houches sur « la Physique de la Matière finement divisée), M. Daoud ed. (Springer Verlag) 1985.

[9] Wilkinson, D. and Willemsen, J., J. Phys. A 16 (1983) 3365.

[10] Wilkinson, D. and Barsony, M., J. Phys. A 17 (1984) L129.

[11] Wilkinson, D., Phys. Rev. A 30 (1984) 520.

[12] Mandelbrot, B. B., The Fractal geometry of Nature (Freeman, San Francisco) 1984.

[13] Weitz, D. A. and Oliveira, M., Phys. Rev. Lett. 52 (1984) 1433.

[14] Stauffer, D., Phys. Rep. 54 (1979) 1.

[15] Wilkinson, D., in Proc. de l'Ecole d'Hiver des Houches sur « la Physique de la Matière finement divisée), M. Daoud ed. (Springer Verlag) 1985.

[16] Dullien, F. A. L., Porous media, fluid transport and pore structure (Academic Press, New York) 1979. 